\title{
The Low-power Design of a Storage-type Downhole Pressure Gauge and Its Application

\author{
Liping Wang ${ }^{1, a^{*}}$, Yong Peng ${ }^{2, b}$ and Heng $\mathrm{Wu}^{2, \mathrm{c}}$ \\ ${ }^{1}$ Xijing University, Xi'an, Shaanxi, China \\ ${ }^{2}$ Xi'an Shiyou University, Xi'an, Shaanxi, China \\ awlpggg@163.com, bypeng@xsyu.edu.cn, wuheng@xsyu.edu.cn
}

\section{Keywords: Downhole; Pressure gauge; Power consumption; Pressure; Monitoring}

\begin{abstract}
A storage-type downhole electronic pressure gauge requires long-time recording of downhole pressure and temperature data during fracturing. Battery life is a vital performance index of this type of pressure gauge. Given inherent limitations in battery capacity, reducing power consumption is the main method to promote a reliable pressure gauge electronic system and extend battery life. A low-voltage power supply, low-power peripheral devices, and a dual power module are used to improve the hardware system. Moreover, the dynamic management of power consumption in multiple operating modes and the dynamic adjustment of the operating frequency of the controller are used to improve the software system. In this way, the power consumption of a pressure gauge electronic system can be considerably decreased. System reliability and stability are also promoted, and the test cost is decreased. Downhole testing of the pressure gauge examined in this study shows that the current consumption of the proposed system is decreased by $69 \%$, and the battery life is extended to more than 3.2 times.
\end{abstract}

\section{Introduction}

The storage-type downhole electronic pressure gauge is widely used in oilfield exploration, formation test of development wells, well testing, production testing, tubing conveyed perforation combined with the formation test, and fracturing because of the high sensitivity, precision, and reliability that characterize this pressure gauge. It can precisely detect downhole pressure and temperature, and it excellently aids both well and production testing during oilfield exploration and development [1].

The storage-type downhole electronic pressure gauge is usually applied under high temperature. This pressure gauge is battery powered, so a high temperature resistance of downhole circuits and power batteries is required. The pressure gauge records long-time downhole pressure and temperature data. Electric power cannot be turned off while recording, so battery life is a vital performance index for this gauge. Battery capacity can be extended to increase battery life, but the cost also increases. Moreover, the corresponding expansion in battery size decreases the inner space of the gauge.

The lower power consumption of the electronic system of a storage-type downhole electronic pressure gauge can extend battery life and the battery replacement cycle of users. As a result, the test cost can be reduced. Moreover, with the reduction of power consumption, the heat generated by components is also reduced, and the lifetime of the components is extended. As the current decreases, the interference of electromagnetic radiation and thermal noise both decrease, so system performance is improved [2]. Fracturing is an important measure to increase production in reservoir development. Fracturing is an important process in reservoir reformation technology in oilfield development [3] [4]. In this study, low-power technology is analyzed and studied, and a low-power storage-type downhole electronic pressure gauge for fracturing is designed.

\section{Structural Composition}

Before well fracturing operations, storage-type downhole electronic pressure gauges are set in the 
bottom hole together with fracturing strings to automatically detect and record pressure and temperature data during fracturing. After completion of fracturing operations, the fracturing strings are pulled out, and the pressure gauges are removed. The data in the pressure gauge memory can be downloaded through the data playback system. The collected data during fracturing are replayed, inspected, queried, analyzed, and drawn into a pressure and temperature curve with the use of a management software program. These data serve as the basis for the quantitative analysis in the monitoring of fracturing response [5].

The circuit system of the downhole pressure gauge comprises a pressure sensor, a temperature sensor, a signal processing and amplification module, an analog to digital converter (ADC) module, a microcontroller, a memory module, and a power module. The system uses the single microcontroller as the control core. Fig. 1 shows the diagrams of a 3D sample and an actual pressure gauge. Fig. 2 shows the diagram of the electronic system. The pressure gauge mainly has four parts with high power consumption, i.e., power, sensor, microcontroller, and memory modules.

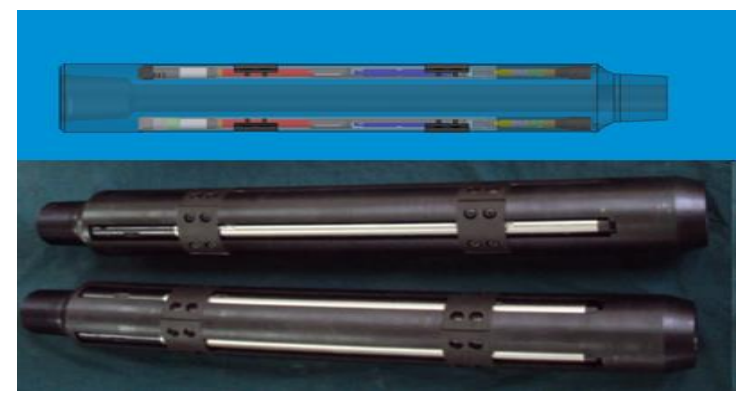

Figure 1. Diagrams of the 3D sample and the actual pressure gauge

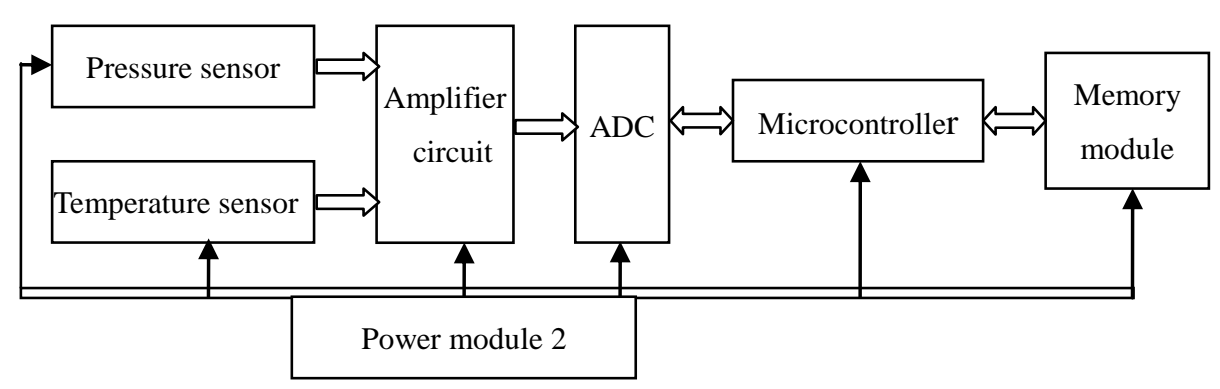

Figure 2. Diagram of the electronic subsystem

\section{Low-power Design of the Pressure Gauge}

Many factors are involved in the low-power design of an electronic system, so new technologies should be considered according to the basic low power consumption principle. With the use of specific applications, appropriate technical indices should be determined flexibly to achieve low power consumption. With the characteristics of the practical operation of a storage-type downhole electronic pressure gauge considered, the main method to achieve low power consumption for a pressure gauge is optimizing the software system and hardware system simultaneously.

Selection of Low-power Components. The selection of components is a crucial factor in a low-power design. The overall power consumption of the system can be significantly decreased through the selection of integrated components with low power consumption [6] [7]. The following components are mainly considered in the system design.

In the normal operations of a pressure gauge, the power consumption of the pressure sensor is a key index. Many types of high-temperature pressure sensors exist, such as the sputtered thin-film pressure transducer and the silicon-on-sapphire high-temperature pressure transducer, with a bridge circuit differential operation. The power supply contains a constant current source or voltage source. The pressure sensor with a constant current supply requires a steady current of $3-5 \mathrm{~mA}$, which 
increases power consumption. A MIDA pressure sensor imported from Russia is used in the system. Because of its high input impedance, the current can be reduced to $0.25 \mathrm{~mA}$ with a low-voltage supply. The power consumption is significantly reduced as a result.

Ultra-low-power microcontroller MSP430 produced by Texas Instruments (TI) is selected. The computer chip can adapt to various requirements of ultra-low voltage and power consumption through the intelligent management of the operating modes of different modules and CPU states [5]. A low power voltage and working current characterize the controller. The active-mode working current is $7 \mu \mathrm{A}$, and the current in standby mode is only $0.8 \mu \mathrm{A}$.

A rail-to-rail out instrumentation amplifier with ultra-low power consumption (INA333) is used. It amplifies the signals before ADC. INA333 operates with a power supply as low as $1.8 \mathrm{~V}( \pm 0.9 \mathrm{~V})$, and the quiescent current is only $133 \mu \mathrm{A}$ with a voltage supply of $3.3 \mathrm{~V}$. Hence, the gauge can achieve low power consumption.

The system uses a low dropout, an adjustable linear regulator with ultra-low power consumption and a maximum output current of $100 \mathrm{~mA}$. The quiescent current under the maximum output current is $335 \mu \mathrm{A}$, and the standby current is only $1 \mu \mathrm{A}$ at $210^{\circ} \mathrm{C}$.

Structure of the Dual Power Module. The storage-type downhole electronic pressure gauge is battery powered and operates automatically in a well. The operation environment and modes are distinctive. Single power is a common structure of current pressure gauges used for fracturing. Batteries are installed before the gauge descends into a well, and then the entire circuit is supplied. Operation parameters, including data sampling and storing frequency, are set on a host computer. The installed pressure gauge is then sent to a well site. During actual test operations, the pressure data should be monitored in real time, whereas the temperature data are measured only when data recording and storage are needed. In the temperature test module, the temperature sensor uses a thermistor PT1000, which requires a current of $3.3 \mathrm{~mA}$ under a power of $3.3 \mathrm{~V}$, so high power consumption results.

To reduce the overall power consumption of the system, two power supply modules are used in the circuit design. Module 1 operates continuously with an output voltage of $3.3 \mathrm{~V}$, so that the microprocessors, pressure sensor, and the amplifier of the sensor can operate to monitor changes in pressure. Moreover, module 2 produces $3.3 \mathrm{~V}$ for the temperature sensor, amplifier, and memory. The operation and standby modes of module 2 are controlled by the microcontroller. Fig. 3 shows a structure diagram of the pressure gauge system with dual power modules.

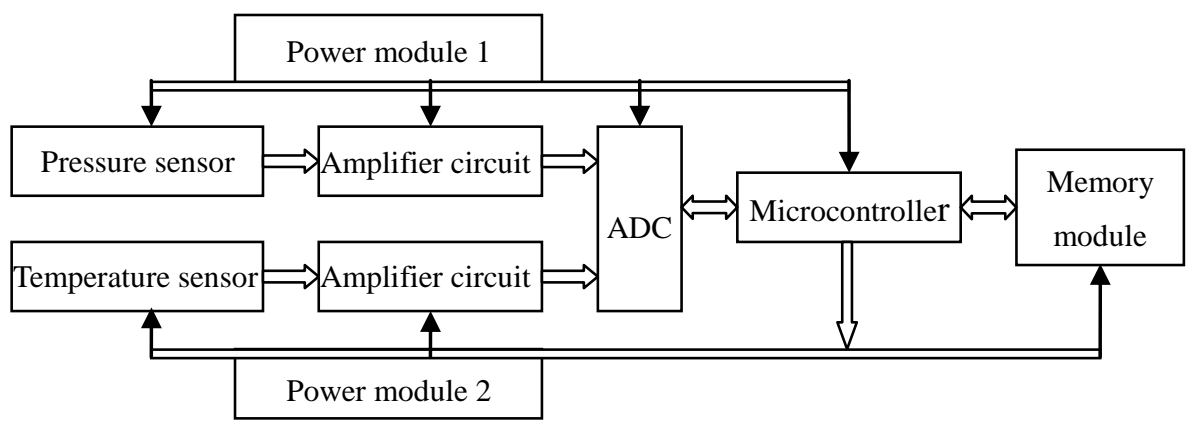

Figure 3. Structure diagram of the pressure gauge with dual power modules

Dynamic Management of Power Consumption. Pre-sampling and the storage mode are commonly used for present storage-type downhole electronic pressure gauges. The operating mode is fixed once the instrument is placed in a well. In practical applications, after a pressure gauge is placed in the well, fracturing takes about $2-3 \mathrm{~h}$ or less. The bottom hole pressure before and after fracturing basically remains unchanged. In addition, given the operation condition and environmental factors, the actual fracturing operation is always inconsistent with the plan and thus results in a long downhole standing time of the gauge. With these operation characteristics considered, the dynamic management of power consumption should be implemented in a 
low-power design. System power supply, data collection, and the data storage state can be managed to reduce the power consumption of the system effectively. The operation efficiency of the system can also be significantly augmented by the promotion of data storage efficiency, which can reduce testing cost.

With the control of the microcontroller, the pressure gauge uses three types of operating modes, i.e., pressure monitoring mode, intermittent measure-storage mode, and continuous measure-storage mode. After power is supplied, the pressure gauge operates in the pressure monitoring mode, and only power module 1 is working. The controller collects pressure signals with a sampling frequency of $1 \mathrm{~Hz}$ and monitors the changes in these signals. The controller also compares the collected pressure data with the last sampling point data. If the difference among these data is less than the preset differential pressure, the system maintains the original operating condition. In the pressure monitoring mode, the power module 2 starts automatically every $4 \mathrm{~min}$ for $100 \mathrm{mS}$. During this period, temperature data are collected, and a record that includes pressure and temperature data is stored.

If the difference between the pressure collected currently and that of the last sampling point is larger than the preset differential pressure in the monitoring mode, power module 2 starts for $100 \mathrm{mS}$. The gauge then transforms into the intermittent measure-storage mode to collect the temperature data. A record that includes pressure and temperature data is stored. If the measured differential pressure is larger than the preset one for $4 \mathrm{~s}$ continuously, the system considers that the fracturing operation has already started. The controller then sends starting signals to wake up power module 2, which supports the temperature sensor, amplifier, and memory. Meanwhile, the sampling frequency of the pressure gauge is changed to $4 \mathrm{~Hz}$. The pressure gauge works in the continuous measure-storage mode. Temperature signals are then collected by the system and stored together with the pressure data.

After the use of the dynamic management mode of power consumption, the integrity of the fracturing data can be guaranteed, and the power consumption of the system is considerably reduced. Table 1, shows the working current and relative power consumption in different operating modes with a test period of 4 min.

Table 1 Working current in power dynamic management mode

(Test condition: $\mathrm{VCC}=3.3 \mathrm{~V}, \mathrm{f}_{\mathrm{SMCLK}}=1 \mathrm{MHz}$ )

\begin{tabular}{|c|c|c|c|c|}
\hline $\begin{array}{l}\text { Operating } \\
\text { Mode }\end{array}$ & Operating Module & $\begin{array}{c}\text { Working } \\
\text { Current }[\mathrm{mA}]\end{array}$ & $\begin{array}{c}\text { Operating } \\
\text { Duration[min] }\end{array}$ & $\begin{array}{c}\text { Relative Power } \\
\text { Consumption }\end{array}$ \\
\hline \multirow{2}{*}{$\begin{array}{l}\text { Monitoring } \\
\text { Mode }\end{array}$} & $\begin{array}{l}\text { Power module } 1 \text {, controller and } \\
\text { pressure measurement }\end{array}$ & 2.5 & 4 & \multirow{2}{*}{$34.6 \%$} \\
\hline & $\begin{array}{l}\text { Power module } 2, \text { memory and } \\
\text { temperature measurement }\end{array}$ & 5.1 & 0.1 & \\
\hline \multirow{2}{*}{$\begin{array}{l}\text { Intermittent } \\
\text { Mode }\end{array}$} & $\begin{array}{c}\text { Power module } 1, \text { controller and } \\
\text { pressure measurement }\end{array}$ & 2.5 & 4 & \multirow{2}{*}{$40.8 \%$} \\
\hline & $\begin{array}{l}\text { Power module } 2, \text { memory and } \\
\text { temperature measurement }\end{array}$ & 5.1 & 0.4 & \\
\hline \multirow{2}{*}{$\begin{array}{l}\text { Continuous } \\
\text { Mode }\end{array}$} & $\begin{array}{c}\text { Power module } 1 \text {, controller and } \\
\text { pressure measurement }\end{array}$ & 2.5 & 4 & \multirow{2}{*}{$100 \%$} \\
\hline & $\begin{array}{l}\text { Power module } 2, \text { memory and } \\
\text { temperature measurement }\end{array}$ & 5.1 & 4 & \\
\hline
\end{tabular}

Dynamic Adjustment of the Microcontroller 's Clock Frequency. System power is directly consumed by the hardware. However, the hardware is driven by the software in practical operations. Different execution and data access instructions directly influence the circuit activities of hardware, so varying actual power consumptions result [8]. The operating mode and operating frequency of a controller significantly affect power consumption. A high frequency generates high power consumption [9] [10]. 
Control programs are developed according to the specific operating characteristics of the storage-type downhole electronic pressure gauge. A controller operates in the standby mode when the pressure gauge is in the pressure monitoring mode. A timer is used to interrupt this standby mode every second, and the controller is subjected to a pressure test. After 200ms, the controller reenters the standby mode. The pressure gauge circuit uses a high-speed crystal oscillator of $11.0592 \mathrm{MHz}$. The downhole low-frequency clock is generated with a $1 / 8$ frequency divider to reduce the power consumption of the circuit during downhole operation. When the pressure gauge communicates with the host computer to set up the parameters or download the data over a well, an operating frequency of $11.0592 \mathrm{MHz}$ is used to enhance the download speed of the data.

The corresponding relationship between the current consumption of an MSP430 single microcontroller in the active mode with the system frequency is

$$
I(A M)=I_{0}(A M)[1 M H z] \cdot f(\text { System })[M H z]
$$

where $A M$ indicates that the single-chip computer is in the active mode. After the dynamic adjustment of the clock frequency is conducted, the consumption of a single microcontroller decreases from $3.8 \mathrm{~mA}$ to $0.48 \mathrm{~mA}$.

\section{Application of the Low-Power Pressure Gauges}

After the pressure gauges are debugged, the gauges are subjected to pressurization, standing, and depressurization tests in a laboratory. The average working current of the pressure gauge during standing is $2.5 \mathrm{~mA}$, and that in the continuous measure-storage operating mode during pressure fluctuations is $7.6 \mathrm{~mA}$. The pressure gauges are then subjected to the downhole test. With the fracturing process in the "Li 648-100" well of some oilfields taken as an example, the pressure curve during the fracturing is shown in Fig. 4.

The testing process lasts for $107.8 \mathrm{~h}$. The actual measured downhole pressure and temperature curve are shown in Fig.4. During fracturing, the maximum pressure is $82.8 \mathrm{MPa}$, and the maximum downhole temperature is $80.9^{\circ} \mathrm{C}$.

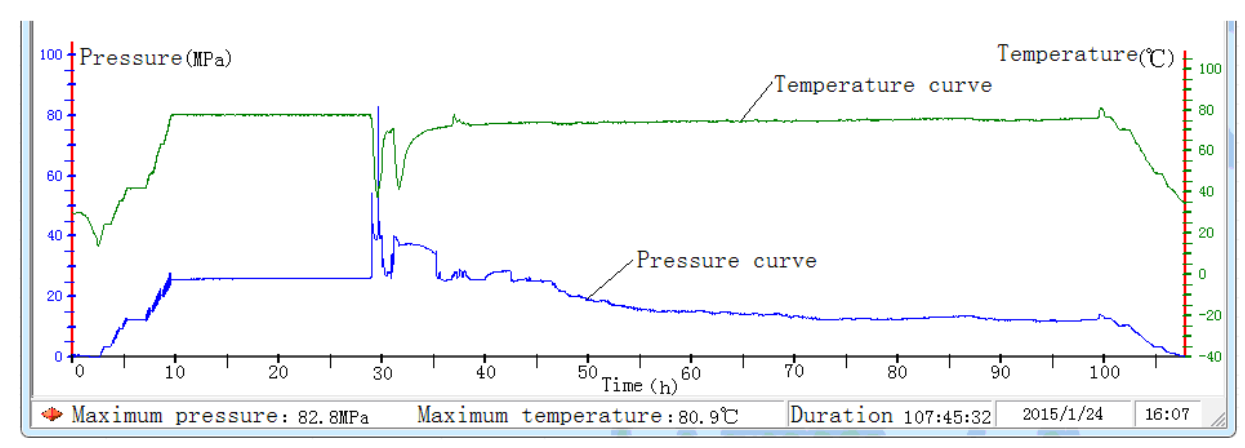

Figure 4. Diagram of the actual measured downhole pressure and temperature curve

Fig.4 depicts that the pressure during the ground standing, downhole standing, and swabbing periods barely changes or changes very slowly. The pressure gauge almost operates in the pressure monitoring mode. Based on the observation of the actual measured data, a new measuring record is stored every $4 \mathrm{~min}$. When the pressure fluctuates sharply during fracturing, the pressure gauge operates in the continuous measure-storage mode. Moreover, when the pressure changes slowly, the gauge operates in the monitoring mode or the intermittent measurement and storage mode.

The downhole testing curve is subjected to time division according to the operating process. The worst condition is analyzed. Suppose that fracturing proceeds for $3 \mathrm{~h}$, and the pressure gauges work in the continuous measure-storage mode during the entire fracturing period. The gauges operate in the intermittent measure-storage mode when the strings are put down and pulled out. In the other time periods, the gauges work in the monitoring mode. 
All the analyses according the real-time data show that the power consumption of a gauge using dual power and dynamic management is reduced by more than $48 \%$ comparing with a single operating mode pressure gauge. Moreover, the pressure gauge use a high input impedance pressure sensor with constant voltage supply, so at least $2.75 \mathrm{~mA}$ current consumption is saved compared with the pressure sensor with a constant current supply. The working current of the system is reduced by $3.32 \mathrm{~mA}$ after application of dynamic clock adjustment for the controller. In conclusion, during the fracturing of "Li 648-100" in Qingcheng, the relative reduction in the current consumption of the circuit is

$$
\left(1-\frac{7.6 \times 48 \%}{5.9+2.75+3.32}\right) \times 100 \%=69 \%
$$

After the aforementioned power reduction methods are used to design the electronic circuit of the pressure gauge. The overall power consumption of the circuit system is significantly reduced, and battery life is extended to over 3.2 times with the same capacity.

\section{Conclusion}

The power consumption of a storage-type downhole electronic pressure gauge is related to various factors. Comprehensively considering factors, such as the actual operating characteristics of the pressure gauge, the technical index, and the economic costs involved, is necessary to reduce system consumption. Appropriate low power consumption technologies are selected to achieve a favorable effect. In the circuit design, the hardware structure is improved with the use of a low-voltage supply, peripheral devices with low power consumption, and a dual power module. The operating mechanism of the software is optimized through methods such as dynamic management of power consumption in multiple operating modes and dynamic adjustment of the operating frequency of the controller. Through these methods, the power consumption of the pressure gauge electronic system is considerably reduced, and battery life is extended. The reliability and stability of the system are promoted, and the testing cost is also reduced. The downhole testing indicates that the current consumption of the pressure gauge is reduced by $69 \%$, and the battery life is extended to more than 3.2 times. In conclusion, the overall performance of the pressure gauge is significantly improved.

\section{Acknowledgments}

This work is supported by the scientific research fund of Xijing University (No. XJ140235).

\section{References}

[1] M.Y. Zhang, Research and Development of Storage-Type Deep Well Electronic Pressure Gauge (Ph.D., Northwestern Polytechnical University, China 2004), p.12. (In Chinese)

[2] Reliability prediction handbook for electronic Equipment(Publishing House of Electronics Industry, China 2007), p.156. (In Chinese)

[3] Q. Zhang, Y.L. Wang and N. Tang, China Petroleum Machinery, Vol. 38(2010) No.(2), p.23. (In Chinese)

[4] Ken Lizak, Drilling \& Completion, SPE(2011) 247-254.

[5] H. Wu, Y. Peng and L.P. Wang, 2013 3rd International Conference on Machinery Electronics and Control Engineering(Jinan, china,2014), 351-355.

[6] S.H. Zhou, Microcomputer Information, Vol. 19(2003) No.(7), p.62. (In Chinese)

[7] B. Bai, A Study on System-Level Low Power Technology (Ph.D., People's Liberation Army Information Engineering University, China 2009), p.48. (In Chinese)

[8] W. Liu, Automation \& Instrumentation, Vol. 24(2009) No.9, P41. (In Chinese) 
[9] Q.X. Yang and R.B. Wang, Electronic Component and Device Applications, Vol. 10(2010) No.(12), p.82.

[10]H.Q. Ren, Fire Control and Command Control, 2008, Vol. 33(2008) No.4, P146. (In Chinese) 